\title{
Expression and selective activation of somatostatin receptor subtypes induces cell cycle arrest in cancer cells
}

\author{
YI ZOU $^{1}$, HAIPING TAN ${ }^{1}$, YUANFENG ZHAO $^{1}$, YUAN ZHOU $^{1}$ and LIN CAO $^{2}$ \\ ${ }^{1}$ Department of Biology, School of Life Science and Technology, Jinan University, Guangzhou, \\ Guangdong 510632; ${ }^{2}$ Department of Anesthesiology of Sun Yat-Sen Memorial Hospital, \\ Sun Yat-Sen University, Guangzhou, Guangdong 510120, P.R. China
}

Received September 29, 2016; Accepted October 18, 2018

DOI: $\quad 10.3892 / 01.2018 .9773$

\begin{abstract}
Somatostatin receptors(SSTRs)areG-protein-coupled plasma membrane receptors that have been determined to be expressed in normal and cancer tissues. Activation of SSTRs frequently results in inhibition of cell proliferation and therefore somatostatin analogues (SSAs) have been used in cancer treatment. However, the variable outcomes of SSA treatment were considered to be the consequences of loss-of-expression of SSTRs and/or subtype-specific effects. In the present study, the patterns of SSTR expression in 160 breast cancer tissues were investigated, and the mechanisms of SSTR activation and the influence on cell proliferation were further characterized. The expression levels of SSTR1-5 were determined using immunohistology. Hemagglutinin-SSTR1 and MYC-SSTR4 were transiently overexpressed in MDA-MB-435S cells, and the potential receptor dimerization was determined using immunofluorescence and co-immunoprecipitation. The influence of SSTR1 and SSTR4 expression/activation on cell proliferation was monitored using flow cytometry. The results demonstrated that all five SSTR subtypes were expressed at variable levels in tumor tissues, with the highest positive expression instance being determined for SSTR1 and SSTR4, with positive expression levels in 90.0 and $71.3 \%$ of tumor tissues, respectively. Immunofluorescence and co-immunoprecipitation revealed SSTR1/SSTR4 heterodimerization, which was increased in response to receptor activation using the subtype-specific SSA L-803087. The translocation of SSTR1/SSTR4 dimers into the cytoplasm upon receptor activation was also observed. Additionally, it was identified using flow cytometry that co-expression and activation of SSTR1 and SSTR4 in MDA-MB-435S cells resulted in a
\end{abstract}

Correspondence to: Dr Lin Cao, Department of Anesthesiology of Sun Yat-Sen Memorial Hospital, Sun Yat-Sen University, 107 Yanjiang Street West, Guangzhou, Guangdong 510120, P.R. China

E-mail: caolin@mail.sysu.edu.cn

Key words: somatostatin receptor, somatostatin analogues, breast cancer, receptor dimerization, cell cycle arrest decreased proportion of S-phase cells. The results of the present study revealed that SSTR1 and SSTR4 are the most frequently expressed SSTR subtypes in breast cancer, and that the cell cycle arrest was mediated by SSTR1/SSTR4 dimerization/activation.

\section{Introduction}

Somatostatin (SST) receptors (SSTRs) are G-protein-coupled plasma membrane receptors with two forms of SST peptides, SS-14 and SS-28, as their natural ligands (1). The two peptides produced by SST cells act as neurotransmitters or paracrine/autocrine regulators, respectively, via five different subtypes of human SSTR (SSTR1-5), encoded by five distinct SSTR genes segregated on chromosomes 14, 16, 17, 20 and 22, respectively (2). Activation of SSTRs frequently results in inhibition of cell proliferation and secretion (3). It is generally accepted that all five SSTR subtypes are involved in the inhibition of the adenylate cyclase-cyclic adenosine 3'5'-monophosphate pathway and stimulate protein tyrosine phosphatases (3). However, a number of effects demonstrated subtype selectivity, and subtype-specific signaling has also been reported $(4,5)$. For instance, SSTR1, 2, 4 and 5 frequently interfere with the mitogen-activate protein kinase pathway to modulate cell proliferation, whereas SSTR3 was indicated to have an increased potential to induce apoptosis $(6,7)$. Additionally, owing to multiple SSTRs being frequently expressed in the same cell, and the existence of ligand-induced dimerization proposed for G-protein-coupled receptors, it is hypothesized that SSTRs are functionally redundant and act in concert $(8,9)$.

Expression levels of SSTRs have been determined in multiple human tissues as well as in the majority of neuroendocrine and non-endocrine tumor types, including hepatocellular carcinoma, pancreatic cancer and breast cancer (10-17). Activation of SSTRs in SSTR-expressing tumors frequently results in marked inhibition of tumor cell proliferation via indirect activities of inhibiting growth hormone secretion and direct activity through SSTR signaling pathways (18). Therefore, SST and SST analogues (SSAs) with improved metabolic stabilities have frequently been used in the treatment of SSTR-positive tumors (19-22). However, the therapeutic results of SSA treatments varied markedly due to the loss-of-expression of SSTRs, different SSTR expression patterns and reasons that are not fully understood (4,23-25). 
In the present study, the expression levels of the five different SSTR subtypes were determined in 160 primary ductal breast tumor samples using immunohistology. All five SSTR subtypes were expressed in the tumor tissues. The expression levels of SSTR1 and SSTR4 were detected in 90.0 and $71.3 \%$ of tumor tissues, respectively. The expression levels of SSTR1 and SSTR4 were determined to be negatively associated with cancer cell differentiation, but were independent of patient age and the cancer stage. SSTR1 and SSTR4 were subsequently overexpressed in cultured MDA-MB-435S cells, which have previously been demonstrated to exhibit decreased endogenous SSTR expression (26). The potential interaction of SSTR1 and SSTR4 was analyzed using immunofluorescence and co-immunoprecipitation. The overexpressed SSTRs were then activated with the subtype-specific SSA L-803087, which has previously been identified to exhibit high selective binding affinity with SSTR1 and SSTR4 (27). The influence of receptor expression and activation on cell proliferation was investigated further using flow cytometry. The results of the present study indicated a ligand-induced heterodimerization of SSTR1 and SSTR4, and the functional significance of the receptor dimerization in regulating cell proliferation. Future investigations on receptor dimerization between other SSTR subtypes and the subsequent effect on cell proliferation will provide valuable references for selection of breast cancer cases suitable for SSA treatment.

\section{Materials and methods}

Breast tumor samples and the clinical information. Sections of all breast tumor samples (fixed with $4 \%$ paraformaldehyde in PBS at $4^{\circ} \mathrm{C}$ overnight and embedded in paraffin) were obtained from the Department of Pathology of The First Affiliated Hospital of Jinan University (Guangzhou, China) from January 2010 to December 2015. A total of 160 primary ductal breast cancer cases, confirmed by pathology, were selected. The clinical references including ages of patients, tumor type and steroid receptor expression levels were provided by the Department of Pathology.

Reagents. Dulbecco's modified Eagle's medium (DMEM), PBS and fetal bovine serum (FBS) used in tissue culture were purchased from Invitrogen; Thermo Fisher Scientific, Inc. (Waltham, MA, USA). Additionally, 3,3-diaminobenzidine tetrachloride, 1,4-dithiothreitol (DTT), dimethylsulfoxide, mouse monoclonal anti-hemagglutinin (anti-HA; cat. no. H3663; 1:1,000), protease inhibitor cocktail, penicillin and streptomycin were purchased from Sigma-Aldrich; Merck KGaA (Darmstadt, Germany). Polyethyleneimine (PEI) was purchased from Polyplus-transfection SA (Illkirch, France). L-803087 and Protein A/G PLUS-Agarose were purchased from Santa Cruz Biotechnology, Inc. (Dallas, TX, USA). Blue Range ${ }^{\mathrm{TM}}$ Prestained Protein Molecular Marker, used for SDS-PAGE, was purchased from Pierce; Thermo Fisher Scientific, Inc. Rabbit monoclonal anti-SSTR1 (cat. no. ab137083; 1:500), rabbit monoclonal anti-SSTR2 (cat. no. ab134152; 1:1,000), rabbit monoclonal anti-SSTR3 (cat. no. ab137026; 1:10,000), rabbit polyclonal anti-SSTR4 (cat. no. ab28578; 1:1,000) and rabbit monoclonal anti-SSTR5 (cat. no. ab109495; 1:1,000) were purchased from Abcam
(Cambridge, MA, USA). The horseradish peroxidase (HRP)-conjugated goat anti-rabbit immunoglobulin G (cat. no. TA140003; 1:10,000), HRP-conjugated goat anti-mouse IgG (cat. no. TA130004; 1:20,000) used for western blot analysis were purchased from OriGene Technologies, Inc. (Rockville, MD, USA). The tetramethylrhodamine-conjugated goat anti-rabbit IgG (cat. no. 111-025-003; 1:100) and the fluorescein isothiocyanate-conjugated mouse anti-rabbit IgG (cat. no. 111-095-003; 1:100) used in immunofluorescence were purchased from Jacksons ImmunoResearch Europe, Ltd. (Newmarket, UK). PrimeSTAR DNA Polymerase, EasyTaq DNA Polymerase, EcoRI, XhoI and T4 DNA ligase were purchased from Takara Bio, Inc. (Otsu, Japan). Ethanol, xylene and $\mathrm{KCl}$ were purchased from Guangzhou Chemical Reagent Factory (Guangzhou, China). EDTA, 0.1\% Triton $\mathrm{X}-100$, hematoxylin and glycerol were purchased from Beijing Dingguo Changsheng Biotechnology Co., Ltd. (Beijing, China). MDA-MB-435S cells were purchased from American Type Culture Collection (Manassas, VA, USA) and maintained in our laboratory according to the instruction.

Expression constructs of hSSTRs. Total mRNA was extracted from cultured HeLa cells using TRIzol ${ }^{\circledR}$ (Invitrogen; Thermo Fisher Scientific, Inc.) reagent, according to the manufacturer's protocol. A total of $2 \mu \mathrm{g}$ total RNA in each reaction was reverse-transcribed into cDNA with oligo(dT) primers using Moloney Murine Leukemia Virus Reverse Transcriptase kit (Promega Corporation, Madison, WI, USA). The cDNA of hSSTR1 and hSSTR4 was amplified with PrimeSTAR using the polymerase chain reaction (PCR) with the following primers: hSSTR1 (1,176 bp), sense, 5'-CCGGAATTCGCCACCATGTTCCCCAATGGC ACCG-3', and antisense, 5'-CCGCTCGAGTCAGAGCGT CGTGATCCGG-3'; and hSSTR4 (1,167 bp), sense, 5'-CCG GAATTCGCCACCATGAGCGCCCCCTCGACG-3', and antisense, 5'-CCGCTCGAGTCAGAAGGTGGTGGTCCT GG-3'. PCR was performed in a Peltier Thermal Cycler 200 programmed with an initial denaturation at $98^{\circ} \mathrm{C}$ for $5 \mathrm{~min}$ followed by 35 cycles of denaturation at $98^{\circ} \mathrm{C}$ for $30 \mathrm{sec}$, annealing at $55-58^{\circ} \mathrm{C}$ for $30 \mathrm{sec}$ and extension at $72^{\circ} \mathrm{C}$ for $90 \mathrm{sec}$. The PCR products were verified by electrophoresis on $1 \%$ agarose gel and visualized by ethidium bromide staining under ultraviolet light. The purified PCR products were then digested with EcoRI/XhoI and cloned using T4 ligase into pcDNA6-Myc/His (Invitrogen; Thermo Fisher Scientific, Inc.) and pCMV-HA (Clontech Laboratories, Inc., Mountainview, CA, USA). The constructs were screened using PCR with Taq polymerase and the sequences of the constructs were verified by DNA sequencing (RuibioBiotech, Beijing, China).

Cell culture and transfection. Human cancer cell line MDA-MB-435S was maintained in DMEM, supplemented with $10 \% \mathrm{FBS}, 100 \mathrm{U} / \mathrm{ml}$ penicillin and $100 \mu \mathrm{g} / \mathrm{ml}$ streptomycin in an atmosphere containing $5 \% \mathrm{CO}_{2}$ at $37^{\circ} \mathrm{C}$. Cells were transfected with the indicated constructs using PEI according to the manufacturer's protocol.

Histology and immunohistochemistry. A total of five $6 \mu \mathrm{m}$ paraffin sections were prepared for each sample. The paraffin 
sections of breast tumor tissues were baked in an oven at $65^{\circ} \mathrm{C}$ for $4 \mathrm{~h}$ prior to being deparaffinized in xylene for $20 \mathrm{~min}$ twice at room temperature. The samples were subsequently rehydrated by incubating at room temperature in an ethanol gradient series for $3 \mathrm{~min}$ at each step $(100,95,80,70$ and $50 \%$, and distilled water). The tissue sections were then washed with PBS three times prior to being boiled in a microwave in $0.01 \mathrm{M}$ citric acid ( $\mathrm{pH}$ 6.0) for $20 \mathrm{~min}$. The samples were then washed once with PBS.

For immunostaining, the sections were treated with $3 \% \mathrm{H}_{2} \mathrm{O}_{2}$ for $10 \mathrm{~min}$ at room temperature and blocked with $1 \%$ bovine serum albumin (Thermo Fisher Scientific, Inc.) plus $0.1 \%$ Triton X-100 in PBS for $30 \mathrm{~min}$ at room temperature. The sections were incubated with the indicated primary antibodies (with PBS in blank control) at $4^{\circ} \mathrm{C}$ overnight and then washed with $3 \mathrm{X}$ PBS. The slides were probed with HRP-conjugated secondary antibodies for $1 \mathrm{~h}$ at room temperature and the excess antibody was washed off with PBS. Subsequently, $250 \mu 1$ DAB per section was then added for color reaction. Following an intensive wash with PBS, the slides were counterstained with hematoxylin for $8 \mathrm{~min}$ at room temperature. The expression levels of SSTRs were scored according to the color and number of positive cells (positive cells $<5 \%, 0$; positive cells $5-25 \%, 1$; positive cells $>25-50 \%, 2$; positive cells $>50 \%$, 3; light tea color, 1; yellow-brownish, 2; brown, 3). An overall score of $0-1$ represented negative, a score of $2-3$ points represented + , a score of 4- 6 represented ++ and a score of $>6$ represented +++ . The incidences of SSTR expressions were presented in percentages. The association between SSTR expression levels and other clinical indexes were analyzed using a $\chi^{2}$ test and $\mathrm{P}<0.05$ was used as statistical significance. A colon cancer tissue sample that was confirmed to exhibit positive SSTR1-5 expression was stored in our lab and included in every experiment as a positive control.

Co-immunoprecipitation and western blot analysis. Cultured cells were collected and resuspended in lysis buffer containing $50 \mathrm{mM}$ Tris/HCl (pH 7.4) (Invitrogen; Thermo Fisher Scientific, Inc.), $100 \mathrm{mM} \mathrm{KCl,} 10 \%$ glycerol, $1 \mathrm{mM}$ EDTA, 1\% Triton $\mathrm{X}-100$ and $1 \mathrm{mM}$ DTT and protease inhibitor cocktail. The supernatants were collected by centrifugation at $12,000 \mathrm{x} \mathrm{g}$ for $15 \mathrm{~min}$ at $4^{\circ} \mathrm{C}$. Protein A/G PLUS-Agarose was used for immunoprecipitation, which was conducted according to the Current Protocols in Cell Biology (28). Aliquots $(15 \mu \mathrm{l})$ of protein samples were then separated by SDS-PAGE (12\% gel) and transferred onto a polyvinylidene fluoride membrane (Whatman; GE Healthcare Life Sciences, Little Chalfont, UK). The membrane was then blocked in 5\% skimmed milk in PBS plus $1 \%$ Triton $\mathrm{X}-100$ for $1 \mathrm{~h}$ at room temperature. Specific primary antibodies and HRP-conjugated secondary antibodies were then used for probing at room temperature for $1 \mathrm{~h}$. The immunoblots were visualized by chemiluminescence with an enhanced chemiluminescent kit (GE Healthcare Life Sciences) and the results were further analyzed with AlphaEase FC software 4.1.0 (Protein Simple, San Jose, CA, USA). Densitometry was performed using a FluorChem ${ }^{\mathrm{TM}}$ system (Protein Simple).

Immunofluorescence analysis of cultured cells. The cultured MDA-MB-435S cells were plated on coverslips and transfected using $16 \mu \mathrm{g}$ PEI per $4 \mu \mathrm{g}$ of pCMV-HA-SSTR1 and/or
pcDNA-MYC-SSTR4. At 24 h post-transfection, the SSTR1 and SSTR4 subtype-specific SSA L-803087 was added into the culture medium to a final concentration of $10 \mathrm{nM}$ (DMEM was added as mock treatment in control cells). The cells were maintained for an additional $24 \mathrm{~h}$ prior to being analyzed. The cells were rinsed twice with PBS and fixed with $3.5 \%$ paraformaldehyde in PBS for $15 \mathrm{~min}$ at room temperature. The cells were subsequently permeabilized or not with $0.2 \%$ Nonidet-P40 in PBS, followed by staining for $1 \mathrm{~h}$ with the appropriate dilutions of the indicated primary antibodies at room temperature, according to the manufacturer's protocol. Excess primary antibodies were washed off with PBS and attached antibodies were then detected with appropriate dilutions of HRP-conjugated secondary antibodies for $1 \mathrm{~h}$ at room temperature, according to the manufacturer's protocol. The coverslips were inverted and mounted onto glass slides for visualization under the 505, 595 and $400 \mathrm{~nm}$ wavelengths on a Zeiss LSM710 confocal microscope (Carl Zeiss AG, Oberkochen, Germany). The images were obtained using a Zeiss LSM 510 EMTA camera (Carl Zeiss AG) and processed with Zeiss LSM Image Browser 4.0 (Zeiss GmbH, Jena, Germany). A total of five different views of co-transfected cells were randomly selected and the dimerization between SSTRs was calculated with Pearson's correlation using Volocity 6.11 (PerkinElmer, Inc., Waltham, MA, USA). Results are presented as the mean \pm standard deviation (SD) and the P-values were determined with Student's t-test.

Flow cytometry and cell cycle analysis. In total, $1 \times 10^{5}$ MDA-MB-435S cells were used to inoculate $1 \mathrm{ml}$ culture medium per well in 24-well Petri dishes. The cells were transfected with pCMV-HA-SSTR1 and/or pcDNA-MYC-SSTR4, and at 24 h post-transfection, the SSTR subtype-specific SSA, L-803087, was added into the culture medium, whereas PBS was added in the mock treatment. The cells were maintained in the aforementioned cell culture conditions for an additional $24 \mathrm{~h}$ prior to further analysis. The cells were then trypsinized and resuspended in fresh medium followed by flow cytometry analysis. For the cell cycle assay, cells were fixed with $70 \%$ ethanol on ice for $30 \mathrm{~min}$. The cells were then suspended in PBS and treated with RNase A (final concentration $100 \mu \mathrm{g} / \mathrm{ml}$; Beijing Solarbio Science \& Technology Co., Ltd., Beijing, China) at $37^{\circ} \mathrm{C}$ for $30 \mathrm{~min}$. Following removal of RNase A by washing once with PBS, the cells were stained with propidium iodide (PI) at $5 \mu \mathrm{g} / \mathrm{ml}$ for $15 \mathrm{~min}$ at room temperature and the cell cycle phases were determined with flow cytometry using a FACSAria I instrument (BD Biosciences, Franklin Lakes, NJ, USA). The data were further analyzed using Modfit LT 4.1 (Verity Software House, Inc., Topsham, ME, USA). The results were obtained from three replicas for each experiment and are presented as the mean \pm SD. P-values were determined with Student's t-test.

\section{Results}

Expression levels of SSTRs in human breast cancer. Paraffin sections of 160 surgically removed human breast-infiltrating ductal carcinoma tumor tissues were collected from the Department of Pathology of The First Affiliated Hospital of 


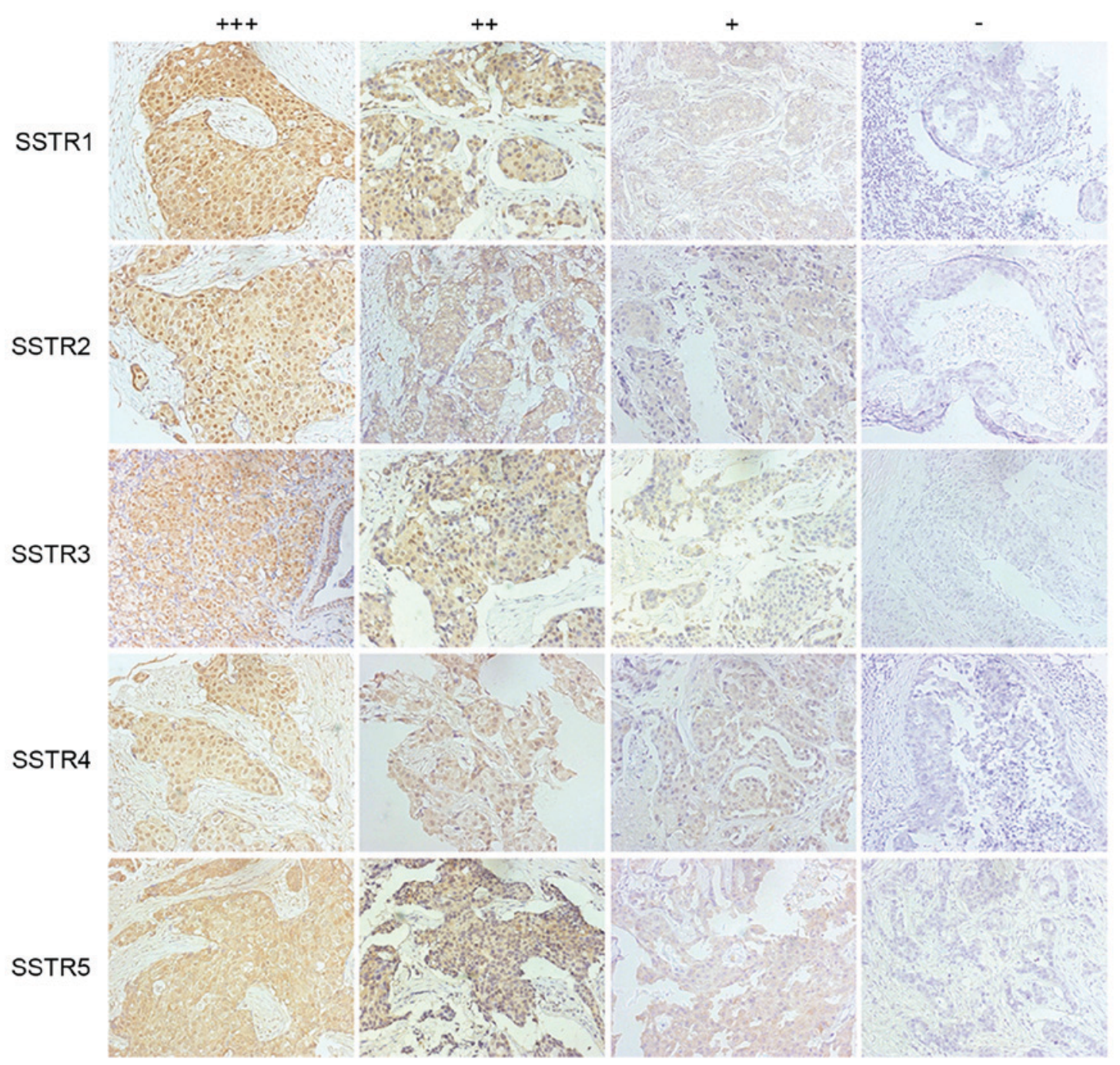

Figure 1. Expression of SSTR1-5 in primary breast tumor tissue. The expression of SSTR1-5 was determined using immunohistology. The variable expression levels of SSTR1-5 were scored according to the number of positive cells and the color of the stain. A colon cancer tissue that was observed with positive SSTR1-5 expression was stored and included in every experiment as a positive control. Magnification, x600. SSTR, somatostatin receptor.

Jinan University. The clinical information, including the patient age, cell differentiation and the expression levels of the relevant molecular markers, was provided by the Department of Pathology of The First Affiliated Hospital of Jinan University. Among these 160 breast cancer tissue samples, the cancer cells were determined to be poorly differentiated in 83 samples, moderately differentiated in 54 samples and well-differentiated in 23 samples, according to the Scarff-Bloom-Richardson system recommended by World Health Organization (29). Histological information of hormone receptor [estrogen receptor (ER), progesterone receptor (PR) and human epidermal growth factor receptor-2] expression levels was provided by the Department of Pathology of The First Affiliated Hospital of Jinan University and was available for 46 samples. The expression levels of the five SSTRs were determined for each sample using immunohistology and their associations with the clinical indexed were further analyzed (data not shown).

All five SSTRs were determined to be expressed at variable levels in breast cancer tissues (Fig. 1), and the expression levels of SSTR1-5 were detected in 90.0, 34.4, $41.9,71.3$ and $44.4 \%$ of cancer tissues, respectively (Table I). The expression levels of these SSTRs were determined to not be associated with the ages of patients. Different subtypes of SSTRs were frequently determined to be co-expressed in tumor tissues. Only 5 samples were observed to be negative for all five subtypes of SSTR expression. A total of 19 cases expressed just one subtype of SSTR, and, among them, 18 were SSTR1-positive and only 1 case expressed SSTR5 alone. The positive expression instances of all five SSTRs was negatively associated with cancer tissue differentiation, and the statistical significance $(\mathrm{P}<0.05)$ in these differences was revealed for the expression levels of SSTR1 and SSTR4 in tumor tissues of different differentiations (Table I). Furthermore, high expression levels of SSTR2 and SSTR3 were only observed in poorly differentiated tumor tissues (Fig. 2). No associations of hormone receptor (estrogen, progesterone and erB-2) expression levels with SSTR expression levels were identified (Table II). However, the expression levels of SSTR2, SSTR3, SSTR4 and SSTR5 exhibited an increased frequency of observation in ER/PR-negative tumor cases.

Heterodimerization of SSTRs. Owing to the common features of ligand-induced dimerization for G-protein-coupled receptors, the potential dimerization of the two most commonly 
Table I. Expression of SSTR subtypes in primary breast tumor tissues.

\begin{tabular}{|c|c|c|c|c|c|c|}
\hline Clinical index & No. of cases & SSTR1+ $(\%)$ & SSTR2+ $(\%)$ & SSTR3+ $(\%)$ & SSTR4+ (\%) & SSTR5+ $(\%)$ \\
\hline \multicolumn{7}{|l|}{ Age, years } \\
\hline$<40$ & 23 & $21(91.3)$ & $8(34.8)$ & $9(39.1)$ & $18(78.3)$ & $12(52.2)$ \\
\hline$>40$ & 137 & $123(89.8)$ & $47(34.3)$ & $58(42.3)$ & $96(70.1)$ & $59(43.1)$ \\
\hline \multicolumn{7}{|l|}{$\begin{array}{l}\text { Cancer cell } \\
\text { differentiation }\end{array}$} \\
\hline Poor & 83 & 79 (95.2) & $30(36.1)$ & $39(47.0)$ & $59(71.1)$ & 37 (44.6) \\
\hline Moderate & 54 & $47(87.0)$ & $18(33.3)$ & $20(37.0)$ & 43 (79.6) & $26(48.1)$ \\
\hline Well & 23 & $18(78.3)^{b}$ & $7(30.4)$ & $8(34.8)$ & $12(52.2)^{\mathrm{b}}$ & $8(34.8)$ \\
\hline Total & 160 & $144(90.0)$ & $55(34.4)$ & 67 (41.9) & $114(71.3)$ & $71(44.4)$ \\
\hline
\end{tabular}

${ }^{a}$ Cancer cell differentiation was evaluated according to the Scarff-Bloom-Richardson system recommended by World Health Organization. ${ }^{\mathrm{b}} \mathrm{P}<0.05$. SSTR, somatostatin receptor.

A
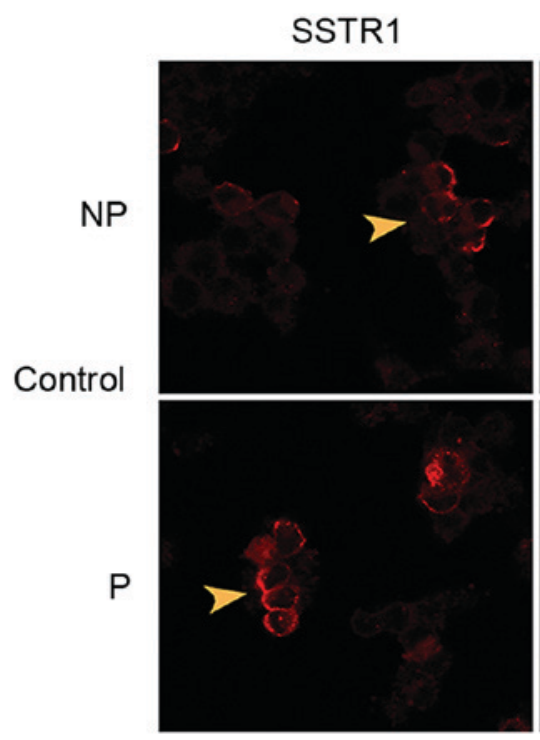

B

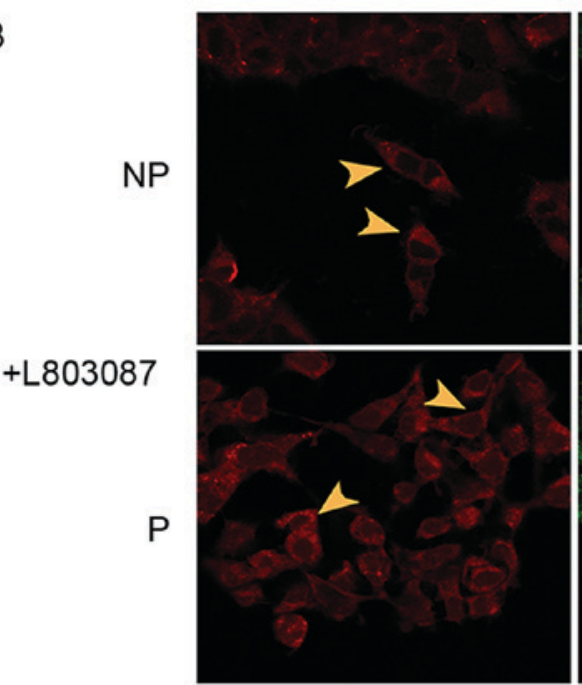

SSTR4
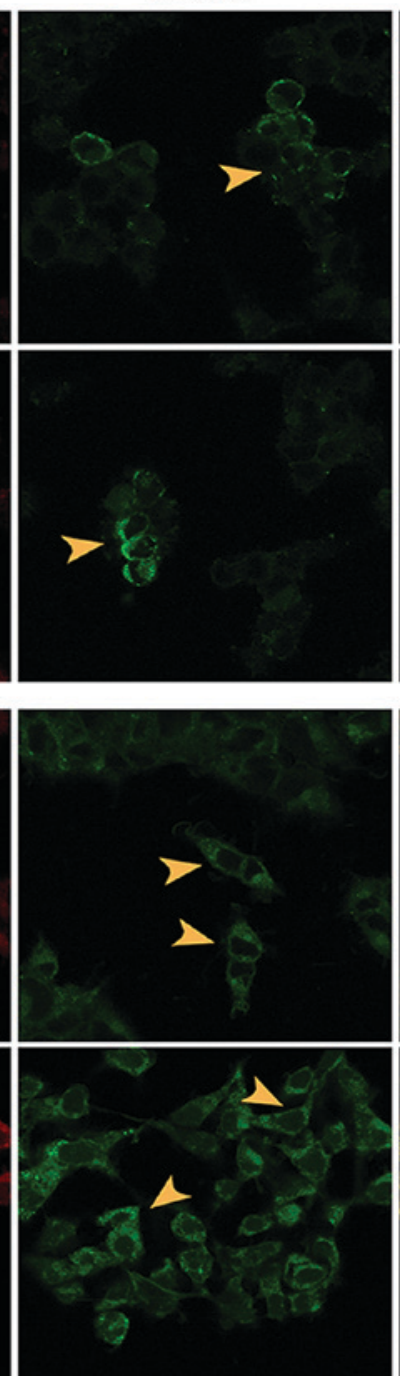

Merged
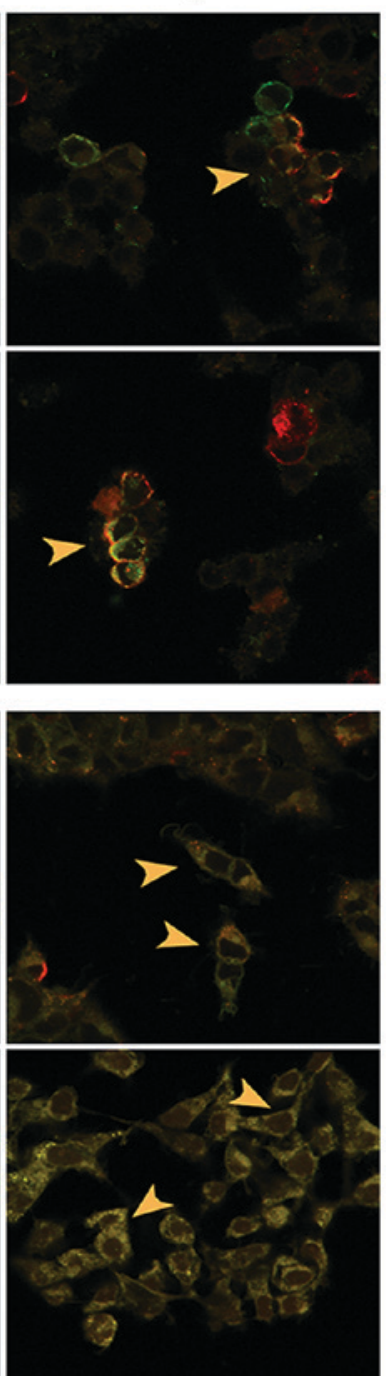

Figure 2. Association of the cellular distributions of SSTR1 and SSTR4. HA-SSTR1 and MYC-SSTR4 were overexpressed in cultured MDA-MB-435S cells. The cellular distributions of SSTRs were displayed by immunofluorescence using confocal microscopy and the potential dimerization was calculated using Pearson's correlation. (A) The cellular distribution of overexpressed SSTR1 (red) and SSTR4 (green), as indicated with arrowheads, in control cells that were not activated using the subtype-specific SSA, L-803087. The cells were non-permeabilized or permeabilized prior to conducting immunofluorescence. (B) The cellular distribution of overexpressed SSTR1 (red) and SSTR4 (green), as indicated with arrowheads, in cells that were activated using the subtype-specific SSA L-803087. The cells were non-permeabilized or permeabilized prior to conducting immunofluorescence. Magnification, x600. SSTR, somatostatin receptor; NP, unpermeablized; $\mathrm{P}$, permeabilized; SSA, somatostatin analogue. 
Table II. Associations of the expression of SSTRs with the expression of hormone receptors.

\begin{tabular}{lcccccc}
$\begin{array}{l}\text { Hormone } \\
\text { receptor expression }\end{array}$ & No. of cases & SSTR1+ $(\%)$ & SSTR2+ $(\%)$ & SSTR3+ $(\%)$ & SSTR4+ $(\%)$ & SSTR5+ $(\%)$ \\
\hline $\begin{array}{l}\text { ER } \\
\text { Positive }\end{array}$ & 31 & $27(87.1)$ & $7(22.6)$ & $4(12.9)$ & $16(51.6)$ & $7(22.6)$ \\
$\quad$ Negative & 15 & $11(73.3)$ & $4(26.7)$ & $3(20.0)$ & $8(53.3)$ & $4(26.7)$ \\
PR & & & & & & \\
$\quad$ Positive & 28 & $24(85.7)$ & $5(17.9)$ & $4(12.9)$ & $14(45.2)$ & $6(19.4)$ \\
$\quad$ Negative & 18 & $14(77.8)$ & $6(40.0)$ & $3(20.0)$ & $10(66.7)$ & $4(26.7)$ \\
$\begin{array}{l}\text { Her-2 } \\
\text { Positive }\end{array}$ & 34 & $28(82.4)$ & $8(23.5)$ & $4(11.8)$ & $17(50.0)$ & $7(20.6)$ \\
Negative & 12 & $10(83.3)$ & $3(20.0)$ & $3(20.0)$ & $7(58.3)$ & $3(20.0)$ \\
\hline
\end{tabular}

The associations of the expression of SSTR1-5 with the expression of hormone receptors were analyzed in 46 breast tumor tissues. Except SSTR1, the probabilities of expression of SSTR2-4 decreased in ER- or PR-positive tumor tissues, although no statistical significance was revealed. ER, estrogen receptor; PR, progesterone receptor; Her-2, human epidermal growth factor receptor-2; SSTR, somatostatin receptor.

expressed SSTRs, SSTR1 and SSTR4, were investigated further. The full-length coding sequences of SSTR1 and SSTR4 were cloned into pCMV-HA and pcDNA6-Myc/His, respectively. Exogenous HA-SSTR1 and MYC-SSTR4 were then co-expressed in cultured MDA-MB-435S cells. No endogenous expression of SSTRs was observed, which was verified using immunoblotting with specific SSTR antibodies (data not shown). The cells were permeabilized or not permeabilized prior to the immunofluorescence assay to primarily display the cytoplasmic or plasma membrane-bound proteins. The target proteins were visualized using confocal microscopy and the potential protein dimerizations were calculated with Pearson's correlation. The heterodimerizations of SSTR1/SSTR4 natively existed on the plasma membrane as well as in the cytoplasm of cells co-expressing HA-SSTR1 and MYC-SSTR4. The Pearson's correlation value of SSTR1 and SSTR4 was significantly increased in permeabilized cells (from $0.74 \pm 0.07$ to $0.89 \pm 0.02$ ) as well as in non-permeabilized cells (from $0.63 \pm 0.04$ to $0.83 \pm 0.03$ ) upon subtype-specific SSA L-803087 activation, compared with non-activated cells. Overexpressed HA-SSTR1 and MYC-SSTR4 were predominantly observed on cytoplasmic membranes prior to activation whereas translocation of activated receptors to the cytoplasm was observed upon ligand induction (Fig. 2).

The dimerization of SSTR1 and SSTR4 was also observed using co-immunoprecipitation. The cell lysates of MDA-MB-435S cells overexpressing HA-SSTR1 and MYC-SSTR4 were immunoprecipitated with an anti-HA antibody. Western blot analysis of the precipitated proteins using an anti-MYC antibody revealed the association between SSTR1 and SSTR4 (Fig. 3A). The heterodimerization of SSTR1 and SSTR4 was significantly increased upon receptor activation using L-803087 (Fig. 3B).

Overexpression and activation of SSTR1/SSTR4 decreases cancer cell proliferation. The influence of SSTR expression and receptor activation on cell proliferation was further investigated in cultured MDA-MB-435S cells using flow cytometry. The cells were transfected with pCMV-HA-SSTR1 and/or pcDNA-MYC-SSTR4, followed by receptor activation using $10 \mathrm{nM} \mathrm{L-803087.} \mathrm{The} \mathrm{control} \mathrm{cells} \mathrm{were} \mathrm{transfected}$ with empty vectors and the mock cells were treated with buffer only. The cell cycles of these cells were subsequently analyzed using flow cytometry. Compared with the control (39.45 $\pm 0.63 \%$ cells in S-phase), the proportion of cells overexpressing SSTR1 or SSTR4 was slightly decreased in S-phase $(38.36 \pm 2.71 \%$; P>0.05; Fig. 4). However, the proportion of S-phase cells overexpressing SSTR1 and SSTR4 was significantly decreased when treated with $10 \mathrm{nM} \mathrm{L-803087}$ (26.06 $\pm 2.79 \%$; $\mathrm{P}<0.05)$. The expression levels of the relevant SSTRs in these cells were verified using western blot analysis (data not shown). The results indicated that the inhibition of cell proliferation was mediated via receptor activation and was subtype-specific.

\section{Discussion}

SST is a natural inhibitory peptide and exerts its anti-secretory/anti-proliferative actions via SSTRs, which have been determined to be ubiquitously expressed in normal and cancer tissues. Owing to the anti-proliferative effects of SSTR signaling, analogs structurally similar to SST that have an increased half-life and are receptor-subtype-selective have been developed and frequently used as a complementary treatment in post-surgical medication for a number of cancer types (1). However, numerous clinical trials reported insensitivity to the treatment with SSA and the lack of benefits was considered to be a consequence of the loss of expression of SSTRs (30). Furthermore, limited information was available on the detailed expression patterns of SSTRs in large clinical trials. Additionally, the underlying molecular mechanisms of receptor activation, and the interplay between SSTRs and other signaling pathways have, to the best of our knowledge, rarely been investigated.

In the present study, the expression pattern of the five SSTR subtypes in 160 primary ductal breast cancer tissues was investigated. The results demonstrated that all five subtypes of SSTR were expressed at variable levels in breast 
A

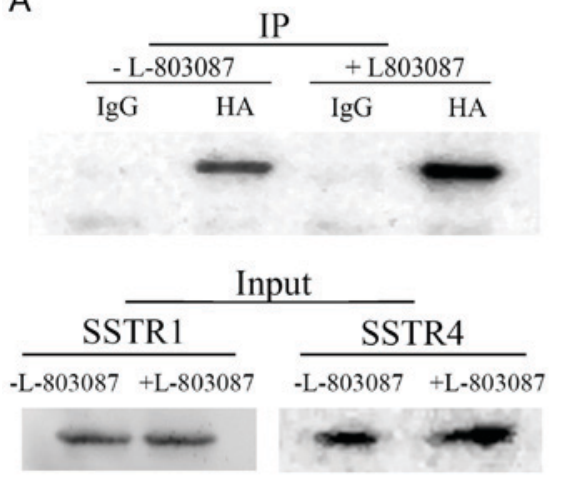

B

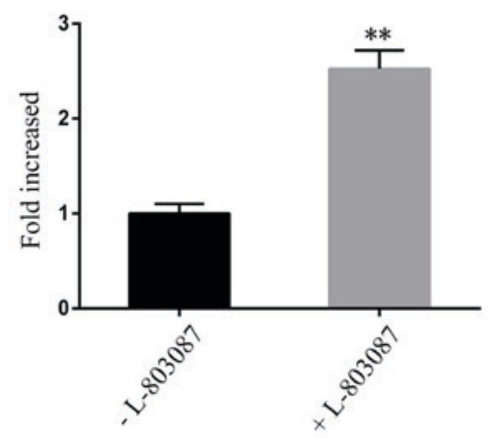

Figure 3. Co-immunoprecipitation of cell extracts overexpressing SSTR1 and SSTR4. (A) Cell lysates were collected from MDA-MB-435S cells overexpressing HA-SSTR1 and MYC-SSTR4. An anti-HA monoclonal antibody was used to immunoprecipitate overexpressed HA-SSTR1 and anti-IgG antibody was used as a negative control. The precipitates were separated by SDS-PAGE (12\% gel) and western blot analysis was performed using an anti-MYC antibody. MYC-SSTR4 was co-precipitated with HA-SSTR1 in control cells (lane 2, upper panel) and in cells treated with L-803087 (lane 4, upper panel). No association was observed in the precipitates using anti-IgG (lane 1 and lane 3, upper panel). The overexpressed HA-SSTR1 and MYC-SSTR4 in the cell lysates were verified by western blot analysis using anti-SSTR1 and anti-SSTR4 monoclonal antibodies, respectively (bottom panel). (B) Densitometric analysis of the western blot analysis using a FluorChem ${ }^{\mathrm{TM}}$ system revealed the significantly increased association between HA-SSTR1 and MYC-SSTR4 in cells activated with the subtype-specific somatostatin analogue. ${ }^{* *} \mathrm{P}<0.01$. SSTR, somatostatin receptor; HA, hemagglutinin; IgG, immunoglobulin G; IP, immunoprecipitation.
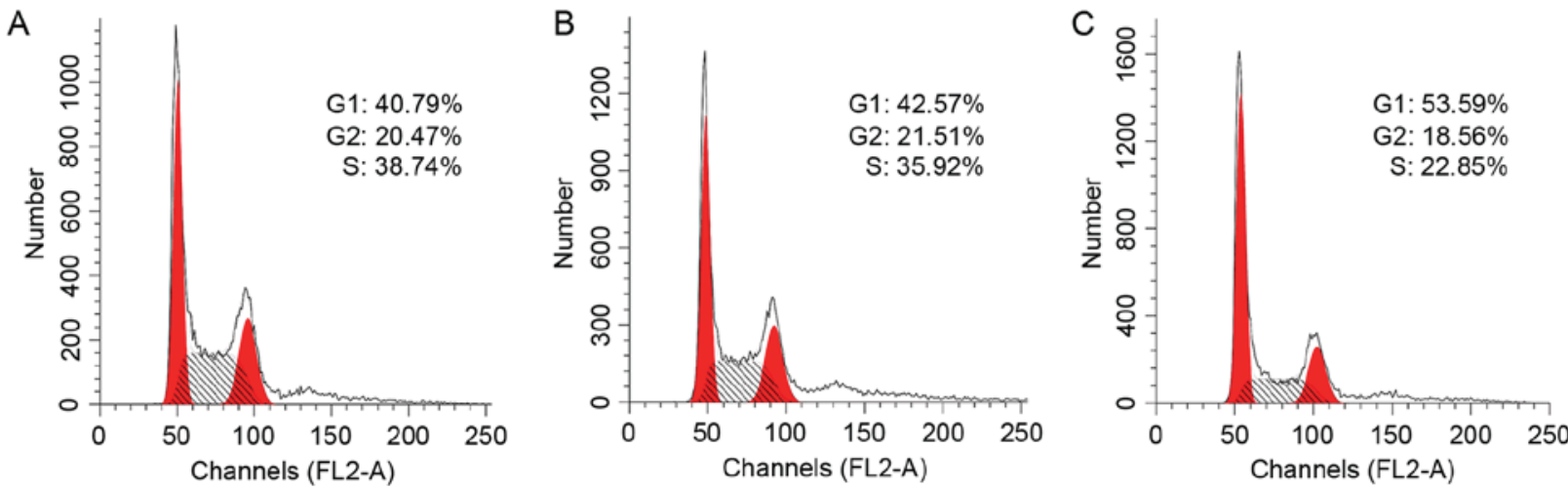

Figure 4. Cell cycle arrest mediated by SSTR1/SSTR4 activation. The cultured MDA-MB-435S cells were transfected with pCMV-HA-SSTR1 and pcDNA6-MYC-SSTR4, followed by activation using subtype-specific SSA L-803087 (10 nM) or not (mock treatment) for an additional $24 \mathrm{~h}$. The control cells were transfected with empty vectors, followed by mock treatment with buffer only. The potential influence of SSTR1/SSTR4 overexpression/activation was analyzed using flow cytometry. (A) Cell cycle phase distribution of MDA-MB-435S cells transfected with pCMV-HA and pcDNA6-MYC/His. (B) Cell cycle phase distribution of MDA-MB-435S cells overexpressing HA-SSTR1 and MYC-SSTR4. (C) Cell cycle phase distribution of MDA-MB-435S cells overexpressing HA-SSTR1 and MYC-SSTR4, followed by treatment with $10 \mathrm{nM} \mathrm{L-803087} \mathrm{for} 24 \mathrm{~h}$. SSTR, somatostatin receptor; HA, hemagglutinin.

cancer tissues. The positive expression instance of SSTR subtypes was highest for SSTR1, followed by SSTR4, SSTR5, SSTR3 and then SSTR2. In contrast with a previous study (31) indicating that SSTR2 and SSTR3 were the most frequently expressed subtypes, the expression levels of SSTR2 and SSTR3 proteins were detected in only 34.4 and $41.9 \%$, respectively, of breast tumor tissues in the present study. Additionally, the expression levels of SSTRs was negatively associated with tumor differentiation and were independent of patient age. The positive expression levels of all five SSTRs were decreased in well-differentiated tumor tissues. Except for SSTR1, the results also indicated that the instances of positive expression of SSTR2-4 increased in ER- or PR-negative cells. This result may not reflect any association between SSTR and ER/PR expression levels, as it may be a coincidence of decreased expression levels of both in poorly differentiated cancer cells. However, it could be a true regulation of SSTR expression by hormone receptors since SST inhibited hormone secretion and tamoxifen/estradiol-differentially regulated SSTR1/2 expression, as reported previously (32). Furthermore, previous studies demonstrated the association of the expression of SSTR subtypes and ER in breast cancer and non-functioning pituitary adenomas $(33,34)$. The detailed regulation of SSTR expression and the potential interplay between SSTR and hormone receptors require further investigation.

Owing to the expression pattern of SSTRs in breast tumor tissues, the potential receptor dimerization between the most frequently expressed SSTR subtypes was further investigated using immunofluorescence. MDA-MB-435S cells were originally characterized as a breast cancer cell line and were later identified to be cross-contaminated with M14 melanoma cells (35). However, owing to their negative endogenous expression of SSTR, MDA-MB-435S cells were selected for the analysis of the potential interaction between SSTR1 and SSTR4 in vitro, to eliminate the interference of other subtypes of endogenous SSTR. Immunofluorescence using confocal microscopy revealed an association of the cellular distribution of overexpressed SSTR1 and SSTR4. 
Additionally, the associated distribution of SSTR1 and SSTR4 was significantly increased upon receptor activation, indicating receptor dimerization as a mechanism of receptor activation. The direct interaction between SSTR1 and SSTR4 was further confirmed using co-immunoprecipitation, which also indicated that the receptor dimerization was notably induced by the subtype-specific SSA L-803087. Furthermore, instead of the predominant plasma membrane-associated distribution, L-803087 induced cytoplasmic translocation of SSTR1/SSTR4, indicating the functional significance of the receptor dimerization. The potential influence of SSA-induced SSTR1/SSTR4 dimerization on cell proliferation was investigated further. Using flow cytometry, the results of the present study indicated that overexpressing SSTR1 and SSTR4 had limited influence on cell proliferation, compared with the control cells transfected with the vector only. However, the proliferation of cells overexpressing SSTR1 and SSTR4 was significantly inhibited upon receptor-subtype-specific SSA, L-803087, activation, indicating that the inhibition of cell proliferation was mediated by receptor dimerization/activation.

Receptor dimerization has been identified to be common for G-protein-coupled receptors (36). Dimerizations have been demonstrated between SSTR subtypes, such as SSTR5 dimerized with SSTR1 and SSTR2, but not SSTR4, as well as between SSTR and other G-protein-coupled receptors, including epidermal growth factor receptor and $\beta_{1}$-adrenergic receptor $(8,20,37-39)$. Owing to the results of the present study and the previous data, it was proposed that the receptor dimerization between SSTR subtypes could be a general mechanism for SSTR signaling $(8,20,37-39)$. Additionally, the cross-talk between SSTR and other associated G-protein-coupled receptors may be critical for SSTRs to confer their cell-type-specific effects. Further investigation of the interaction between other SSTR subtypes and their function will be beneficial for understanding the subtype-specific cellular effects and the mechanisms of SSTR signaling pathways, which will be valuable for optimizing SSA treatment and selection of the patients suitable for SSA treatment.

\section{Acknowledgements}

Not applicable.

\section{Funding}

The present study was sponsored by the National Natural Science Foundation of China (grant no. 81571041) and the Construction Project for the Key Laboratory of Virology of Guangzhou (grant no. 201705030003).

\section{Availability of data and materials}

The datasets used and/or analyzed during the current study are available from the corresponding author on reasonable request.

\section{Authors' contributions}

YZou conceived and designed the study, analyzed and interpreted the data, drafted and revise the manuscript, and approved the final manuscript. HT acquired the data, analyzed and interpreted the data, and drafted the manuscript. YZha and YZho acquired, analyzed and interpreted the data. LC conceived and designed the study, analyzed and interpreted the data and approved the final manuscript. All authors read and approved the final manuscript.

\section{Ethics approval and consent to participate}

Ethics approval was obtained from The Committee of Medical Ethics of the First Affiliated Hospital of Jinan University. Patients provided written informed consent.

\section{Patient consent for publication}

Not applicable.

\section{Competing interests}

The authors declare that they have no competing interests.

\section{References}

1. Günther T, Tulipano G, Dournaud P, Bousquet C, Csaba Z, Kreienkamp HJ, Lupp A, Korbonits M, Castaño JP, Wester HJ, et al: International union of basic and clinical pharmacology. CV. Somatostatin receptors: Structure, function, ligands, and new nomenclature. Pharmacol Rev 70: 763-835, 2018.

2. Day R, Dong W, Panetta R, Kraicer J, Greenwood MT and Patel YC: Expression of mRNA for somatostatin receptor (sstr) types 2 and 5 in individual rat pituitary cells. A double labeling in situ hybridization analysis. Endocrinology 136: 5232-5235, 1995.

3. Florio T: Somatostatin/somatostatin receptor signalling: Phosphotyrosine phosphatases. Mol Cell Endocrinol 286: 40-48, 2008.

4. Zatelli MC, Piccin D, Tagliati F, Bottoni A, Luchin A, Vignali C, Margutti A, Bondanelli M, Pansini GC and Pelizzo MR: Selective activation of somatostatin receptor subtypes differentially modulates secretion and viability in human medullary thyroid carcinoma primary cultures: Potential clinical perspectives. J Clin Endocrinol Metab 91: 2218-2224, 2006.

5. Ruscica M, Magni P, Steffani L, Gatto F, Albertelli M, Rametta R, Valenti L, Ameri P, Magnaghi V and Culler MD: Characterization and sub-cellular localization of SS1R, SS2R, and SS5R in human late-stage prostate cancer cells: Effect of mono- and bi-specific somatostatin analogs on cell growth. Mol Cell Endocrinol 382: 860-870, 2014.

6. Theodoropoulou M and Stalla GK: Somatostatin receptors: From signaling to clinical practice. Front Neuroendocrinol 34: 228-252, 2013.

7. War SA and Kumar U: Coexpression of human somatostatin receptor-2 (SSTR2) and SSTR3 modulates antiproliferative signaling and apoptosis. J Mol Signal 7: 1-15, 2012.

8. Rocheville M, Lange DC, Kumar U, Sasi R, Patel RC and Patel YC: Subtypes of the somatostatin receptor assemble as functional homo- and heterodimers. J Biol Chem 275: 7862-7869, 2000.

9. Missiaglia E, Dalai I, Barbi S, Beghelli S, Falconi M, Della PM, Piemonti L, Capurso G, Di FA and Delle FG: Pancreatic endocrine tumors: Expression profiling evidences a role for AKT-mTOR pathway. J Clin Oncol 28: 245-255, 2010.

10. Vikić-Topić S, Raisch KP, Kvols LK and Vuk-Pavlović S: Expression of somatostatin receptor subtypes in breast carcinoma, carcinoid tumor, and renal cell carcinoma. J Clin Endocrinol Metab 80: 2974-2979, 1995.

11. Skanberg J, Ahlman H, Benjegard SA, Fjalling M, Forssell Aronsson EB, Hashemi SH, Nilsson O, Suurkula M and Jansson S: Indium-111-octreotide scintigraphy, intraoperative gamma-detector localisation and somatostatin receptor expression in primary human breast cancer. Breast Cancer Res Treat 74: 101-111, 2002.

12. Cakir M, Dworakowska D and Grossman A: Somatostatin receptor biology in neuroendocrine and pituitary tumours: Part 1-molecular pathways. J Cell Mol Med 14: 2585-2591, 2010. 
13. Callison JC Jr, Walker RC and Massion PP: Somatostatin receptors in lung cancer: From function to molecular imaging and therapeutics. J Lung Cancer 10: 69-76, 2011.

14. Watt HL, Kharmate G and Kumar U: Biology of somatostatin in breast cancer. Mol Cell Endocrinol 286: 251-261, 2008.

15. Hicks RJ: Use of molecular targeted agents for the diagnosis, staging and therapy of neuroendocrine malignancy. Cancer Imaging 10 Spec no A: S83-S91, 2010.

16. Reubi JC, Schaer JC, Waser B and Mengod G: Expression and localization of somatostatin receptor SSTR1, SSTR2, and SSTR3 messenger RNAs in primary human tumors using in Situ Hybridization. Cancer Res 54: 3455-3459, 1994.

17. Schmid HA, Lambertini C, Van Vugt HH, Barzaghi-Rinaudo P, Schäfer J,Hillenbrand R, Sailer AW, Kaufmann M and Nuciforo P: Monoclonal antibodies against the human somatostatin receptor subtypes 1-5: Development and immunohistochemical application in neuroendocrine tumors. Neuroendocrinology 95: 232-247, 2011.

18. Hofland LJ and Lamberts SW: Somatostatin receptors and disease: Role of receptor subtypes. Baillières Clin Endocrinol Metab 10: 163-176, 1996.

19. Wang S, Bao Z, Liang QM, Long JW, Xiao ZS, Jiang ZJ, Liu B, Yang J and Long ZX: Octreotide stimulates somatostatin receptor-induced apoptosis of SW480 colon cancer cells by activation of glycogen synthase kinase-3beta, A Wnt/beta-catenin pathway modulator. Hepatogastroenterology 60: 1639-1646, 2013.

20. Grant M, Alturaihi H, Jaquet P, Collier B and Kumar U: Cell growth inhibition and functioning of human somatostatin receptor type 2 are modulated by receptor heterodimerization. Mol Endocrinol 22: 2278-2292, 2008.

21. Hasskarl J, Kaufmann M and Schmid HA: Somatostatin receptors in non-neuroendocrine malignancies: The potential role of somatostatin analogs in solid tumors. Future Oncol 7: 895-913, 2011.

22. Raderer M, Hejna MH, Muller C, Kornek GV, Kurtaran A, Virgolini I, Fiebieger W, Hamilton G and Scheithauer W: Treatment of hepatocellular cancer with the long acting somatostatin analog lanreotide in vitro and in vivo. Int J Oncol 16 : $1197-1201,2000$

23. Li M, Zhang R, Li F, Wang H, Kim HJ, Becnel L, Yao Q, Chen C and Fisher WE: Transfection of SSTR-1 and SSTR-2 Inhibits Panc-1 Cell proliferation and renders Panc-1 cells responsive to somatostatin analogue. J Am Coll Surg 201: 571-578, 2005.

24. Arena S, Barbieri F, Thellung S, Pirani P, Corsaro A, Villa V, Dadati P, Dorcaratto A, Lapertosa G, Ravetti JL, et al: Expression of somatostatin receptor mRNA in human meningiomas and their implication in in vitro antiproliferative activity. J Neurooncol 66 : 155-166, 2004

25. Barbieri F, Pattarozzi A, Gatti M, Aiello C, Quintero A, Lunardi G, Bajetto A, Ferrari A, Culler MD and Florio T: Differential efficacy of SSTR1, -2 , and -5 agonists in the inhibition of C6 glioma growth in nude mice. Am J Physiol Endocrinol Metab 297: 1078-1088, 2009

26. Xu Y, Song J, Berelowitz M and Bruno JF: Estrogen regulates somatostatin receptor subtype 2 messenger ribonucleic acid expression in human breast cancer cells. Endocrinology 137: 5634-5640, 1996.
27. Patel YC: Somatostatin and its receptor family. Front Neuroendocrinol 20: 157-198, 1999.

28. Bonifacino JS, Gershlick DC and Dell'Angelica EC: Immunoprecipitation. Curr Protoc Cell Biol 1: 71, 2016.

29. Bansal C, Singh US, Misra S, Sharma KL, Tiwari V and Srivastava AN: Comparative evaluation of the modified Scarff-Bloom-Richardson grading system on breast carcinoma aspirates and histopathology. Cytojournal 9: 4, 2012.

30. Mohamed A, Romano D, Saveanu A, Roche C, Albertelli M, Barbieri F, Brue T, Niccoli P, Delpero JR, Garcia S, et al: Anti-proliferative and anti-secretory effects of everolimus on human pancreatic neuroendocrine tumors primary cultures: Is there any benefit from combination with somatostatin analogs? Oncotarget 8: 41044-41063, 2017.

31. Kumar U, Grigorakis SI, Watt HL, Sasi R, Snell L, Watson P and Chaudhari S: Somatostatin receptors in primary human breast cancer: Quantitative analysis of mRNA for subtypes 1-5 and correlation with receptor protein expression and tumor pathology. Breast Cancer Res Treat 92: 175-186, 2005.

32. Rivera JA, Alturaihi H and Kumar U: Differential regulation of somatostatin receptors 1 and 2 mRNA and protein expression by tamoxifen and estradiol in breast cancer cells. J Carcinog 4: $10,2005$.

33. Nishioka H, Tamura K, Iida H, Kutsukake M, Endo A, Ikeda Y and Haraoka J: Co-expression of somatostatin receptor subtypes and estrogen receptor- $\alpha$ mRNAs by non-functioning pituitary adenomas in young patients. Mol Cell Endocrinol 331: 73-78, 2011.

34. Frati A, Rouzier R, Lesieur B, Werkoff G, Antoine M, Rodenas A, Darai E and Chereau E: Expression of somatostatin type-2 and -4 receptor and correlation with histological type in breast cancer. Anticancer Res 34: 3997-4003, 2014.

35. Rae JM, Creighton CJ, Meck JM, Haddad BR and Johnson MD MDA-MB-435 cells are derived from M14 melanoma cells-a loss for breast cancer, but a boon for melanoma research. Breast Cancer Res Treat 104: 13-19, 2007.

36. Wang W, Qiao Y and Li Z: New insights into modes of GPCR activation. Trends in Pharmacol Sci 39: 367-386, 2018.

37. Somvanshi RK, War SA, Chaudhari N, Qiu X and Kumar U: Receptor specific crosstalk and modulation of signaling upon heterodimerization between $\beta 1$-adrenergic receptor and somatostatin receptor-5. Cell Signal 23: 794-811, 2011.

38. Watt HL, Kharmate GD and Kumar U: Somatostatin receptors 1 and 5 heterodimerize with epidermal growth factor receptor: Agonist-dependent modulation of the downstream MAPK signalling pathway in breast cancer cells. Cell Signal 21: 428-439, 2009.

39. Watt HL and Kumar U: Colocalization of somatostatin receptors and epidermal growth factor receptors in breast cancer cells. Cancer Cell Int 6: 1-19, 2006.

This work is licensed under a Creative Commons Attribution-NonCommercial-NoDerivatives 4.0 International (CC BY-NC-ND 4.0) License. 